\title{
Appropriateness of endoscopy referrals in a district general hospital
}

\author{
Authors: Shuann Shwana ${ }^{A}$ and Dlovan Taha ${ }^{B}$
}

\section{Aims}

Most cancers of the upper gastrointestinal tract are not diagnosed at an early stage; therefore, it is important for GPs to refer patients with suspected symptoms as early as possible. The appropriateness of the indications for oesophagogastroduodenoscopy (OGD) is crucial in referring patients to endoscopy units, improving costeffectiveness and providing better patient care.

The aim of this study was to evaluate the appropriateness of urgent referrals to the endoscopy unit to reduce unnecessary referrals.

\section{Methods}

Over a 4-month period, information was gathered retrospectively on 90 patients referred to the endoscopy unit for urgent OGD. The appropriateness of referrals was established using National Institute for Health and Care Excellence (NICE) clinical guidelines and Welsh cancer standards referrals standards.

\section{Results}

The results showed that $24 \%$ of patients referred for urgent OGD did not meet the referral criteria. Out of those inappropriate referrals, only two referrals were downgraded by the consultant. Of those patients who met the criteria for referral, $93 \%$ were not seen within 7 days as recommended by the guidelines, and $8 \%$ of them waited longer than 28 days to be seen after being referred. Four referred patients $(4.4 \%)$ were diagnosed with adenocarcinoma of the oesophagus and two patients $(2.2 \%)$ were diagnosed with Barrett's oesophagus. The rest had other non-malignant conditions.

\section{Conclusions}

Use of the national guidelines to make appropriate referrals to the endoscopy unit can improve selection of patients for the procedure. However, to avoid missed diagnoses of serious disease, using the guidelines must be tailored to the specific clinical setting.

Authors: ${ }^{\text {APrince }}$ Charles Hospital, Merthyr Tydfil, UK; ${ }^{B}$ University Hospital of Wales, Cardiff, UK

\section{Conflict of interest statement}

None declared. 